\title{
OPTICAL NONLINEARITY IN LIQUID CRYSTALLINE OPTICAL WAVEGUIDES
}

\author{
M.A. Karpierz*, A.W. Domański, M. Sierakowski, M. Świllo \\ AND T.R. WOLIŃSKI \\ Warsaw University of Technology, Institute of Physics \\ Koszykowa 75, 00-662 Warszawa, Poland
}

\begin{abstract}
The analysis of optical orientational nonlinearity in nematic liquid crystalline planar waveguides is presented. The analyzed nonlinearity leads to unique properties that are not observed in other types of nonlinear waveguides. Theoretical results are confirmed by experimental data obtained in liquid crystalline directional couplers and in the self-focusing effect observed in the planar liquid crystalline waveguides.
\end{abstract}

PACS numbers: 42.6.5.Wi, 42.70.Df

\section{Introduction}

Liquid crystals possess many unique physical, optical, and electro-optical properties that cause that they are important materials in numerous technical applications. In the nematic phase, the correlation among liquid crystalline molecules is very strong because of the high anisotropy as well as the collective behavior of the molecules. This is responsible for the fact that liquid crystal molecules can easily reorient even with a very low externally applied field. Additionally, due to a large birefringence and good transparency (from near UV to far IR spectrum), liquid crystals are important anisotropic objects for modern optoelectronics [1].

Generally, nonlinear optical phenomena in liquid crystals arise from molecular reorientation and thermal effects. In this paper a detailed analysis of the nonlinear orientational effect in nematic liquid crystalline waveguides is presented. For this type of nonlinearity the liquid crystal molecules reorient due to interactions with electric field of the light wave which changes the local birefringence axis of liquid crystal. The orientational nonlinearity in nematic liquid crystalline waveguides leads to numerous effects not observed in other types of nonlinearity [2-4], i.e. the threshold and optical bistable effects resulting in nonlinear refractive index changes, strong dependence on light polarisation as well as possibility of controlling the nonlinearity by external electric or magnetic fields. The orientational nonlinearity leads to extremely large nonlinear changes of the refractive index and these

*e-mail: karpierz@if.pw.edu.pl 
changes can be easily achieved for relatively low values of the light power. Theoretically analyzed unique properties of liquid crystalline nonlinear waveguides have been also confirmed in recently reported experiments with liquid crystalline directional couplers [5] and formation of spatial soliton in liquid crystalline planar waveguides [6].

\section{Theory of liquid crystalline waveguides}

At a given temperature nematic liquid crystal molecules fluctuate around the mean direction defined by the director $n$. The distortion of the molecular alignment corresponds to the free energy density [1]

$$
f_{\mathrm{F}}=\frac{1}{2} K_{11}(\operatorname{div} n)^{2}+\frac{1}{2} K_{22}(n \cdot \operatorname{rot} n)^{2}+\frac{1}{2} K_{33}(n \times \operatorname{rot} n)^{2},
$$

where $K_{i i}$ are elastic constants for three different deformations: splay $(i=1)$, twist $(i=2)$, and bend $(i=3)$. External electric or magnetic fields create a torque on the molecules and change the mean orientation of the liquid crystal. For the optical frequencies the interaction with the magnetic field can be neglected and the light wave interacts with the nematic liquid crystal through the electric field. The orientation-dependent term in the interaction energy density between the electric field and the liquid crystal molecules is equal to

$$
f_{\mathrm{opt}}=-\frac{\varepsilon_{0} \Delta \varepsilon}{2}\left\langle(n \cdot E)^{2}\right\rangle,
$$

where $\Delta \varepsilon=\varepsilon_{\|}-\varepsilon_{\perp}, \varepsilon_{\|}=n_{\mathrm{e}}^{2}$, and $\varepsilon_{\perp}=n_{\circ}^{2}$ are, respectively, extraordinary and ordinary components of the electric permittivity of the nematic liquid crystal. The total free energy density composed of the deformation energy $f_{\mathrm{F}}$ and the interaction energy $f_{\text {opt }}$ fulfils a minimization procedure, which leads to the Euler-Lagrange equations.

It is convenient to introduce the orientation angle $\theta$ between the director $n$ and the $x$-axis. The angle $\theta$ is sufficient to describe molecular reorientation limited to two dimensions. In this case the liquid crystal director $n$ at the boundaries and the electric field vector lie in the same plane. We assume the waveguide configurations as in Fig. 1 and then $n=(\cos \theta, 0, \sin \theta)$. Consequently, the guided TM modes with $E_{x}$ and $E_{z}$ components of the electric field are taken into account. This type of guided waves does not change the type of the analyzed configuration in the nonlinear case while the hybrid modes (with the $E_{y}$ component of electric field) will tend to reorient liquid crystal molecules perpendicularly to the $x z$ plane. Assuming that the electric field amplitude in the $z$ direction is homogeneous, the Euler-Lagrange equations take the following form [3]:

$$
\begin{gathered}
\frac{\partial^{2} \theta}{\partial x^{2}}\left(K_{33} \cos ^{2} \theta+K_{11} \sin ^{2} \theta\right)-\frac{1}{2}\left(\frac{\partial \theta}{\partial x}\right)^{2}\left(K_{33}-K_{11}\right) \sin 2 \theta+\frac{\partial^{2} \theta}{\partial y^{2}} K_{22} \\
+\frac{\varepsilon_{0} \Delta \varepsilon}{4}\left[\left(E_{x} E_{z}^{*}+E_{x}^{*} E_{z}\right) \cos 2 \theta+\left(\left|E_{z}\right|^{2}-\left|E_{x}\right|^{2}\right) \sin 2 \theta\right]=0,
\end{gathered}
$$

which describes reorientation of the liquid crystal due to the electric field of the electromagnetic wave. The electric field induced reorientation is the field of the TM guided mode with the following components:

$$
E_{x}, H_{y}, E_{z} \propto \exp \left(\mathrm{i} \omega t-\mathrm{i} k_{0} N z\right),
$$




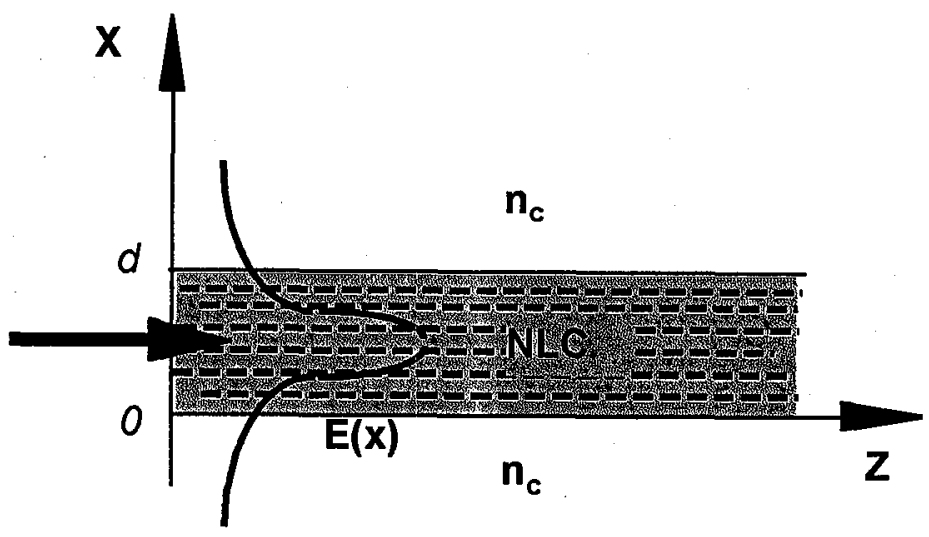

Fig. 1. Schematic draws of the analyzed liquid crystalline waveguide.

where $N$ is an effective refractive index of the guided mode, which fulfils Maxwell's equations

$$
\begin{aligned}
\frac{\mathrm{d}}{\mathrm{d} x} E_{z} & =\mathrm{i} k_{0}\left(Z H_{y}-N E_{x}\right), \\
\frac{\mathrm{d}}{\mathrm{d} x} H_{y} & =\mathrm{i} k_{0} \frac{1}{Z}\left(\varepsilon_{x z} E_{x}+\varepsilon_{z z} E_{z}\right), \\
\varepsilon_{x x} E_{x} & =Z N H_{y}-\varepsilon_{x z} E_{z},
\end{aligned}
$$

where $Z=\mu_{0} c, \varepsilon_{i j}(i, j=x, y, z)$ are components of the electric permittivity tensor

$$
\varepsilon=\left(\begin{array}{ccc}
\varepsilon_{\perp}+\Delta \varepsilon \cos ^{2} \theta & 0 & \Delta \varepsilon \sin \theta \cos \theta \\
0 & \varepsilon_{\perp} & 0 \\
\Delta \varepsilon \sin \theta \cos \theta & 0 & \varepsilon_{\perp}+\Delta \varepsilon \sin ^{2} \theta
\end{array}\right) .
$$

Reorientation of the liquid crystal changes the birefringence axis orientation and it means that the light modifies the electric permittivity tensor. The described mechanism is the basis of the giant orientational nonlinearity observed in liquid crystals. Since the dielectric anisotropy is relatively large (even $\Delta \varepsilon \approx 0.6$ ) this nonlinearity creates very large refractive index changes.

\section{Properties of nonlinear guided waves}

Nonlinear properties of liquid crystalline waveguides strongly depend on the initial orientation of molecules, which is a consequence of their interaction with the surrounding surfaces at the boundary of the cell. First we assume the initial planar orientation of the liquid crystal, i.e. the boundary conditions $\theta(0)=$ $\theta(d)=\pi / 2$ and consequently the initial orientation without any external fields is homogeneous: $\theta(x)=\pi / 2$. The theoretical results are calculated for the $6 \mathrm{CHBT}$ (4-trans-4'-n-hexyl-cyclohexyl-isothiocyanatobenzene) nematic liquid crystal acting as the film and characterized by the extraordinary and ordinary refractive indices equal to $n_{\mathrm{e}}=1.52$ and $n_{\circ}=1.69$, respectively. 
Figure 2 presents the orientation angle $\theta$ distribution for different values of the light power guided in the fundamental nonlinear TM mode in a waveguide with the liquid crystalline layer with the thickness $d=2 \mu \mathrm{m}$ and for the equal refractive indices of the substrate and the cover $n_{\mathrm{c}}=1.45$. As a guided power $P$ the dimensionless parameter normalized to the dielectric anisotropy and the elastic constant of the liquid crystals as well as the thickness of the waveguide film were used. For the $10 \mu \mathrm{m}$ diameter of the light beam in the planar waveguide the unity of the used guided power $P$ correspond approximately to $20 \mathrm{~mW}$ of light power.

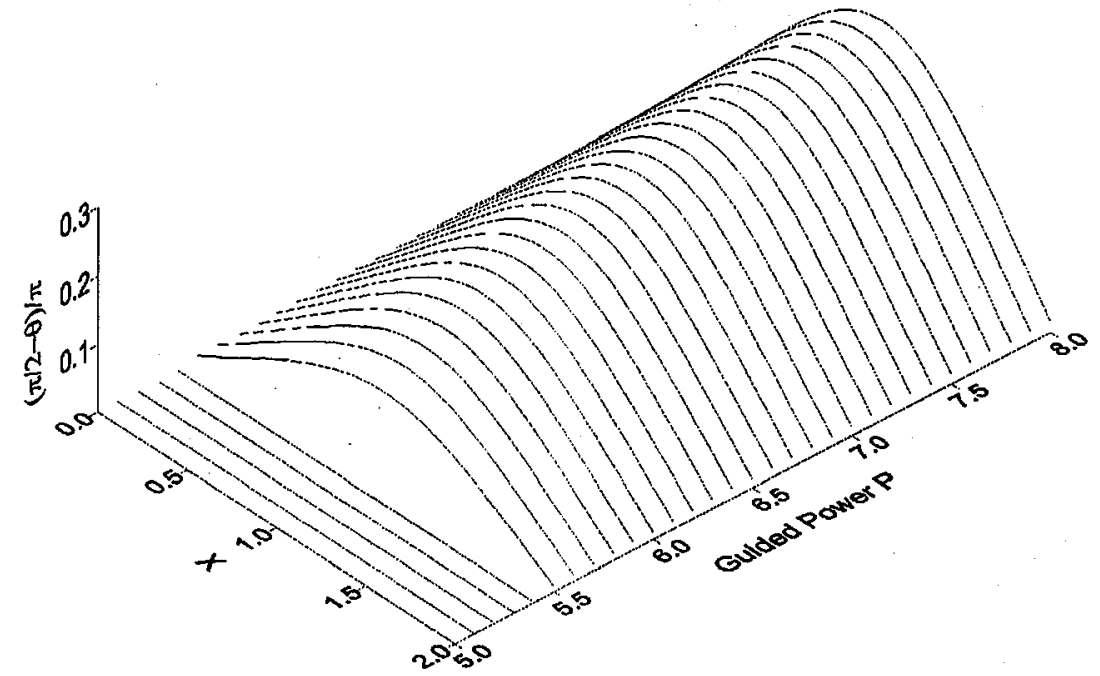

Fig. 2. Reorientation angle $\theta$ distribution for different values of the light power in the guided mode for the waveguide with the film thickness $d=2 \mu \mathrm{m}$ and refractive index of the surrounding covers $n_{c}=1.45$.

Figure 2 shows that for a guided power lower than $P \approx 5.6$ the liquid crystal has the initial orientation and any nonlinear reorientation does not occur. The reorientation appears above a certain threshold value of the light power. This phenomenon exists in liquid crystals when the electric field tends to reorient liquid crystalline molecules at an angle $\pi / 2$ and it is called the threshold Frederiks transition effect. Figure 3 presents changes of the effective refractive index of the guided mode due to optical reorientation (dashed curve corresponds to the effect from Fig. 2). The threshold transition effect produces discontinuous changes of the effective refractive index and it can be a source of optical bistability of the guided mode (see Fig. 3 for different values of refractive index in the waveguide cover). The changes in the refractive index due to the reorientation are very large and hence the guided mode profile changes drastically. These changes could be large enough to support the reorientation even for lower powers than the light power value, which is necessary to induce reorientation. Therefore, the bistability of the effective refractive index characteristics is obtained. The reorientation effect depends not only on the local value of the electric field but also it depends on the electric field profile in a cross-section. On the other hand, molecular reorientation 


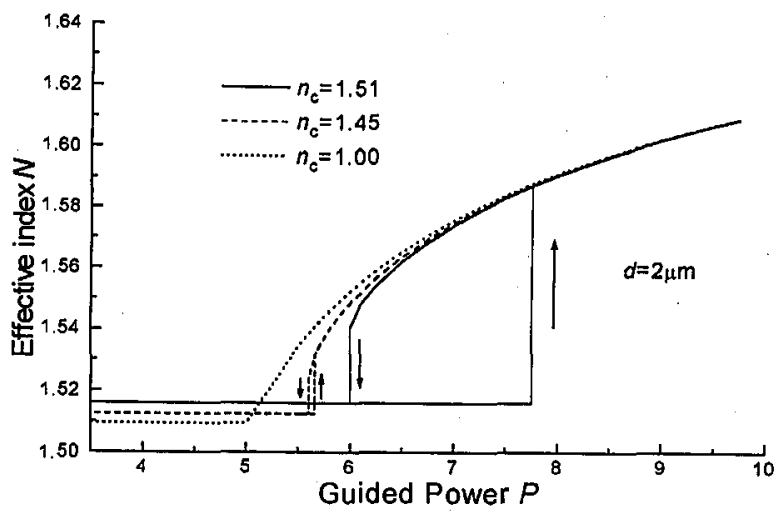

Fig. 3. Effective refractive index changes versus the light power guided in the nonlinear fundamental TM mode for different values of the refractive index in the cover $n_{c}$. The dashed curve corresponds to the behavior presented in Fig. 2.

changes the electric field profile in guided electromagnetic waves. This means that the reorientation nonlinear effect has a non-local nature and it is a source of a feedback necessary in optical bistability. It should be noted that for the classically analyzed Kerr-type nonlinearity (i.e. the refractive index is linearly dependent on the local value of the light power density $P: n=n_{1}+n_{2} P$ ) the optical bistability in waveguide modes is predicted only in the case when the waveguide cover is nonlinear.

The optical bistability in the guided mode is connected with the threshold Frederiks effect and in configurations without threshold phenomena the optical bistability disappears. This is observed in liquid crystalline waveguides with initially tilted planar orientation, i.e. for the boundary conditions $\theta(0)=\theta(d)=$ $\pi / 2-\theta_{0}$ and consequently for the initial orientation without any external fields: $\theta(x)=\pi / 2-\theta_{0}$ (Fig. 4a). In these waveguides the bistability does not exist even for a very weak tilt $\theta_{0} \ll 1$ (Fig. $4 \mathrm{~b}$ ).
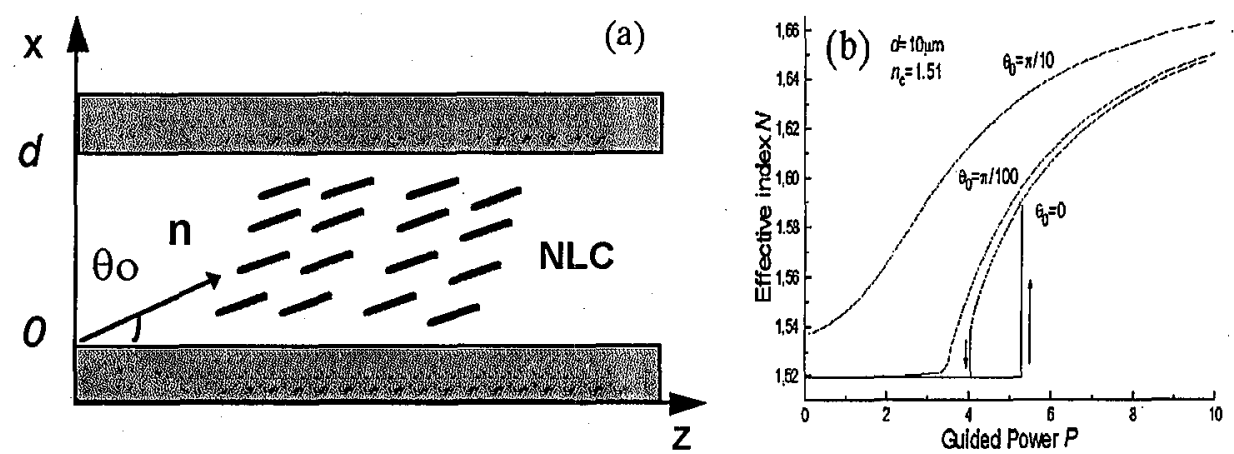

Fig. 4. Geometry of the liquid crystalline waveguide with tilted planar orientation (a) and effective refractive index changes for different values of the initial tilt $\theta_{0}$ (b). 
The nonlinear effect in liquid crystalline waveguides can be modified not only by modifying the configuration of the layer but also by the external constant or low frequency electric (or magnetic) fields. This external field can support or diminish reorientation and therefore, it can force or suppress the orientational nonlinearity. Figure 5 presents the effective refractive index changes for different values of the electric field supporting reorientation. Any increase in this field causes decreasing in the threshold value of the reorientation and hence reduces the optical bistability. Above the critical value $E_{\text {th }}$ of the electric field reorientation starts without any threshold.

Optical nonlinear effects in liquid crystalline waveguides also depend on the light polarization. The influence of the light polarization on the optical nonlinear-
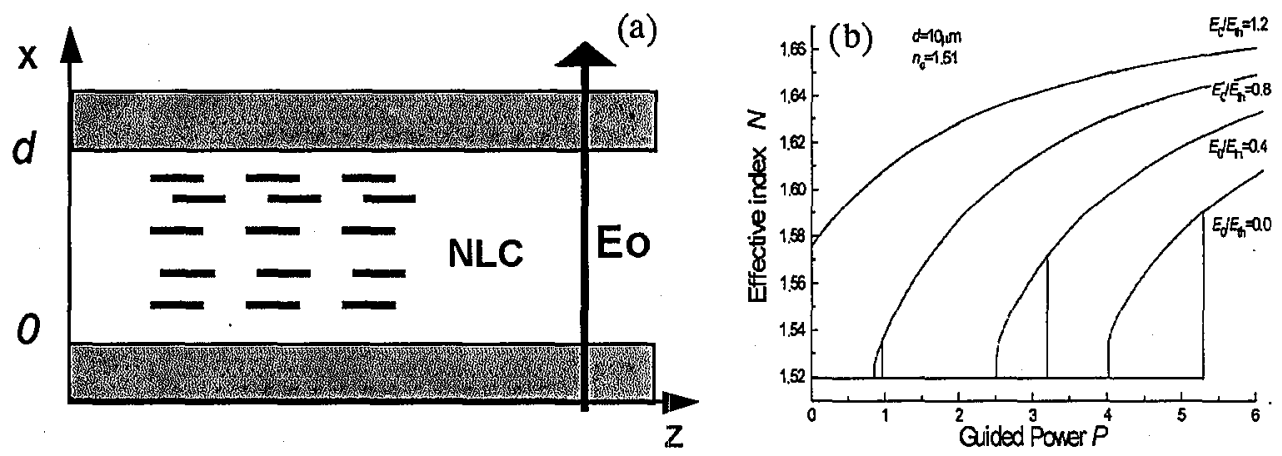

Fig. 5. Geometry of the liquid crystalline waveguides with external electric field $E_{0}$ supporting reorientation (a) and the obtained in this configuration effective refractive index characteristics (b) for different values of electric fields $E_{0} / E_{\text {th }}$, where $E_{\text {th }}$ is the threshold value of the reorienting field.

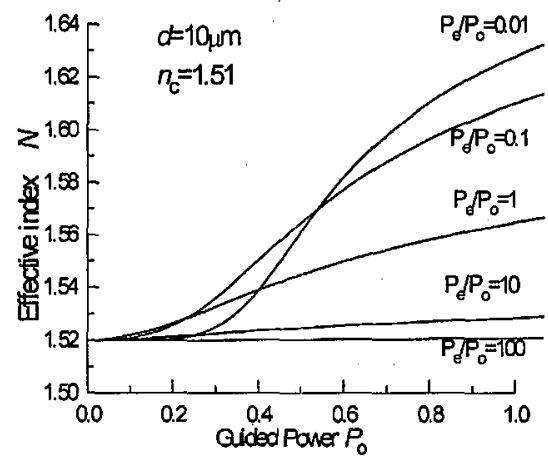

Fig. 6. The effective refractive index $N$ changes of the TE guided mode versus the light power for different ratios of the power guided in the TE mode $P_{\mathrm{o}}$ (with the $E_{y}$ component of the electric field) and the power of light $P_{\mathrm{e}}$ guided simultaneously in the TM mode (with the strong $E_{x}$ component of the electric field). Both modes are in the same phase and the nematic liquid crystal has a homeotropic alignment. 
ity in liquid crystalline waveguide can be shown in the homeotropically aligned liquid crystalline film, i.e. with the boundary conditions $\theta(0)=\theta(d)=0$ and consequently for the initial orientation $\theta(x)=0$. In this configuration the fundamental TM mode does not induce any reorientation and the waveguide is linear. On the other hand, the TE-like mode with a strong $E_{y}$ electric field component will create nonlinearity analogous to that analyzed above (for planar orientation and TM modes). In the presence of both mode polarizations the nonlinearity is dependent on the ratio of powers guided in TM $\left(P_{\mathrm{e}}\right)$ and TE-like $\left(P_{\mathrm{o}}\right)$ modes. Figure 6 shows the effective refractive index changes of the TE-like mode versus the guided light power for different ratios between power in the TM and TE-like modes. Calculations were done for the low-nonlinearity approximation that is valid in the absence of threshold phenomena. In the case of a stronger TM mode the obtained nonlinear reorientation appears only in the neighborhood of the planes where both modes are in the same phase.

\section{Experimental results}

The unique properties of the orientational nonlinearity in liquid crystalline waveguides were tested experimentally during the investigation of the self-focusing phenomena in planar waveguides and in the directional coupler structure $[5,6]$. Figure 7 shows the configuration for observation of the self-focusing effect. The homeotropically aligned nematic liquid crystal 6CHBT with a thickness $d=10 \mu \mathrm{m}$ surrounded by the glass plates played the role of the waveguide film. The light from the semiconductor laser at $\lambda=842 \mathrm{~nm}$ was coupled to the film through a single-mode fiber. The scattering of the beam in the liquid crystalline waveguide was observed by a CCD camera. The obtained cross-sections of the detected light for the light power guided in the waveguide $P \approx 30 \mathrm{~mW}$ is presented in Fig. 8 .

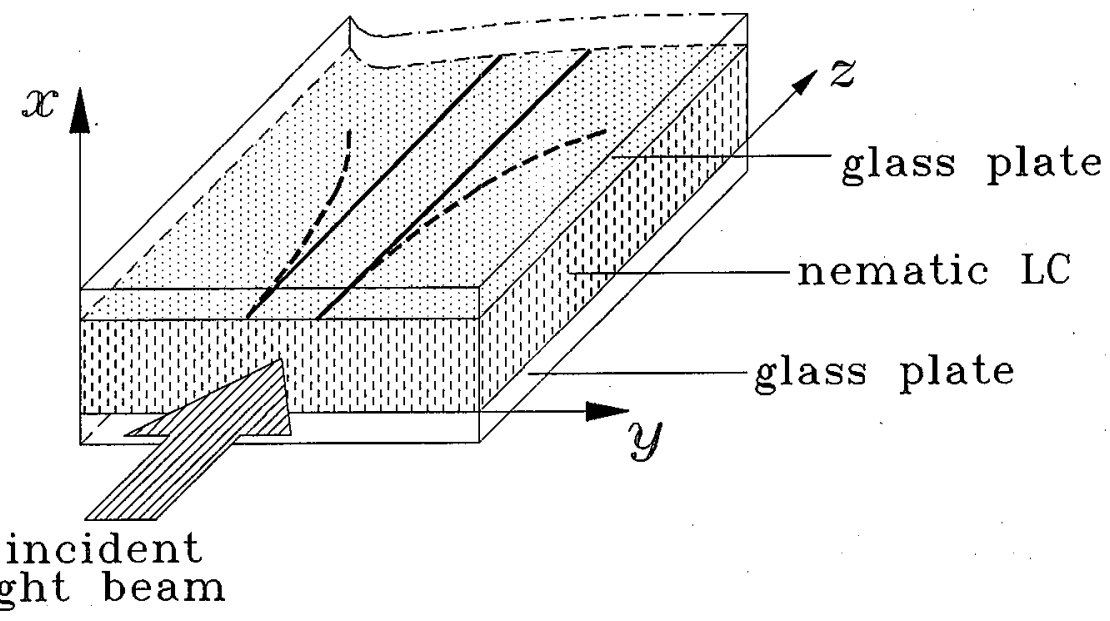

Fig. 7. The schematic drawing of propagation of the laser beam in the planar liquid crystalline waveguide. 

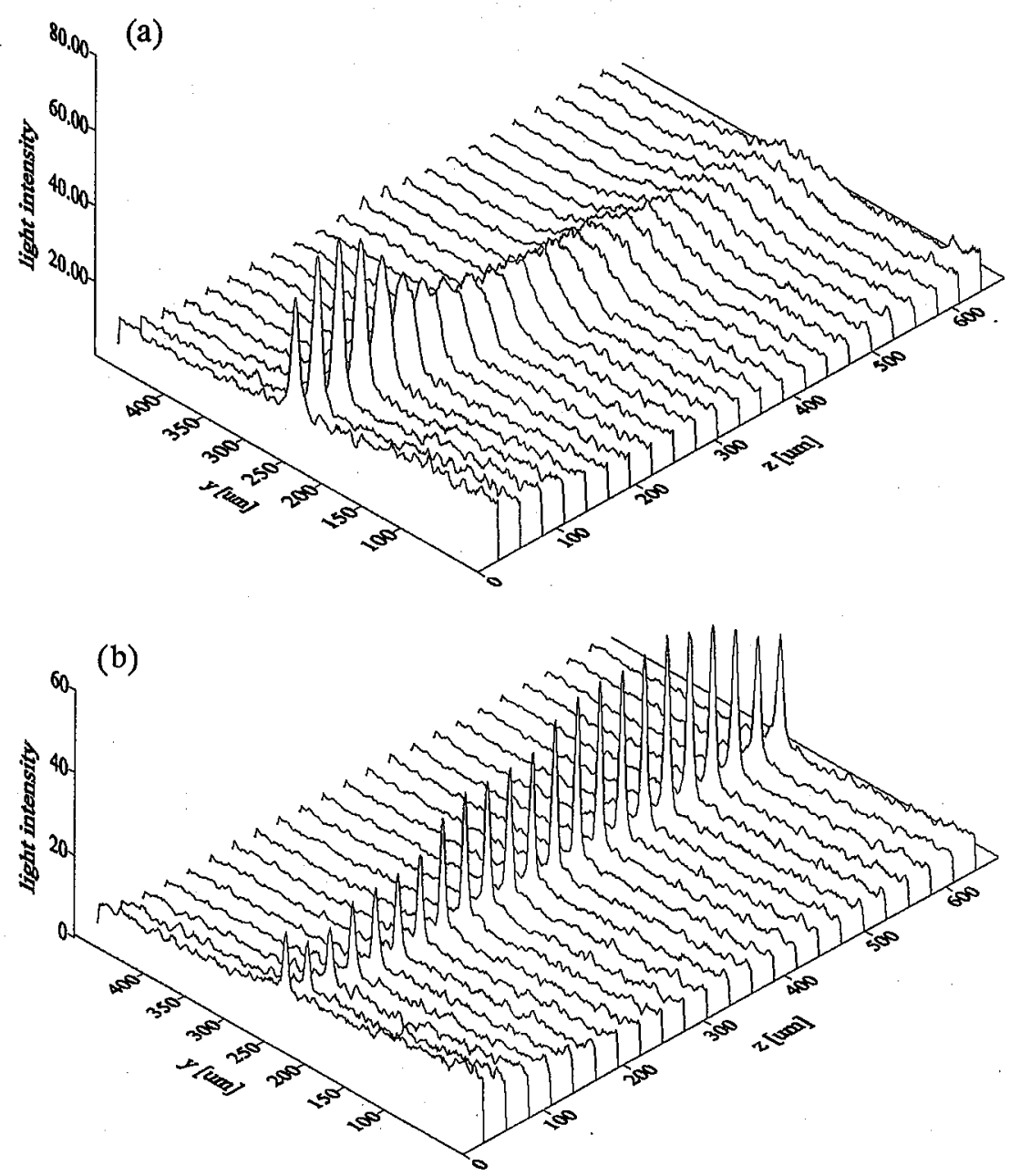

Fig. 8. The cross-section distribution of the light intensity of the laser beam guided in nonlinear liquid crystalline waveguide for the TE-like polarization (a) and the TM-like polarization of the light (b). The results were obtained experimentally for $30 \mathrm{~mW}$ of light power at a wavelength $\lambda=842 \mathrm{~nm}$ for the 6 CHBT liquid crystal waveguide with a. thickness $d=10 \mu \mathrm{m}$.

The self-focusing effect in planar waveguides appears as a stable one only when the nonlinearity is not stronger than the square function (Kerr-type nonlinearity) $[7,8]$. Therefore, in the analyzed liquid crystalline waveguide stable self-focusing can be obtained only for the TM-like polarized light beam. For the TE-like polarized light (Fig. 8a) the beam is initially focused and then it spreads larger than in the linear diffraction case. However, for the same light power but for another polarization (TM-like) self-focusing becomes stable and the spatial soliton is observed (Fig. 8b). 

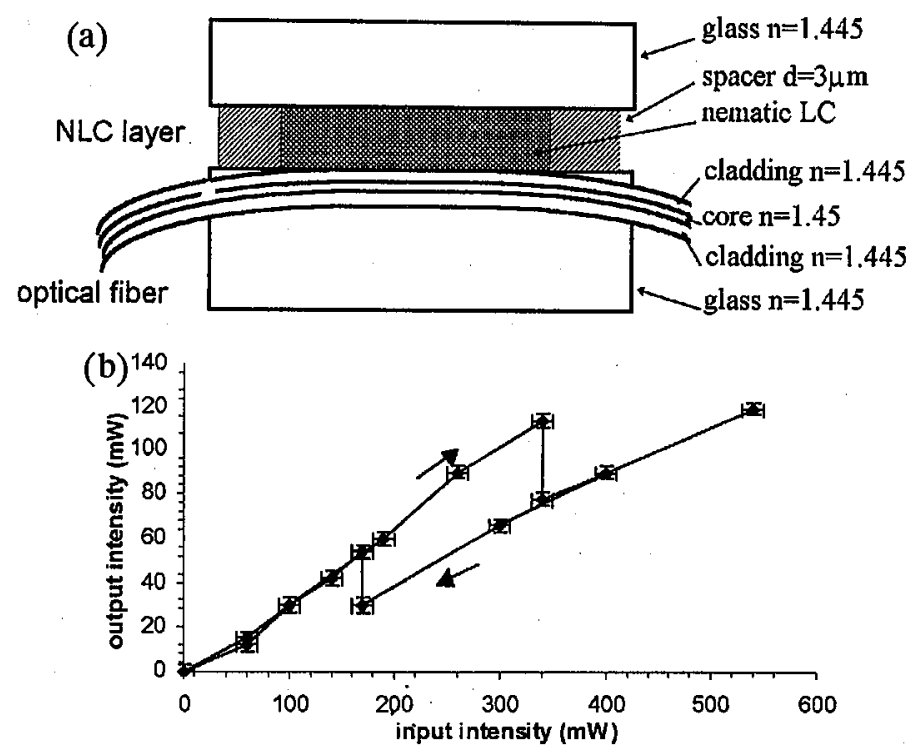

Fig. 9. Liquid crystalline directional coupler (a) and its observed experimentally transmission characteristics (b) for the argon laser with a wavelength $\lambda=514 \mathrm{~nm}$.

Optical bistability in liquid crystalline waveguide structures was observed in configuration with the directional coupler. The schematic drawing of the investigated fiber-to-planar waveguide directional coupler is shown in Fig. 9a. The coupling between waveguides in the directional coupler depends on effective refractive indices of separated waveguides. For a nonlinear coupler the increasing of the light intensity changes the refractive indices and consequently the transmission between waveguides depends on the input light intensity. In a classical Kerr-type nonlinearity the optical bistability effect is not observed since any optical hysteresis can exist only in systems with a feedback, which is absent in directional couplers with the Kerr-type nonlinearity. A typical input/output characteristic for the liquid crystalline directional coupler is plotted in Fig. 9b. As a light source the argon laser at $\lambda=514 \mathrm{~nm}$ was used and the output light was detected at the optical fiber end. The stable optical loop with a contrast of about $40 \%$ was observed for an input intensity of $\sim 340 \mathrm{mlW}$.

\section{Conclusions}

In this paper, the experiment as well as theoretical analysis of nonlinear properties of nematic liquid crystalline waveguides were presented. The orientational nonlinearity in liquid crystals has different unique properties, which can be very useful in all-optical switching and data processing systems due to very low power necessary to observe nonlinear effects. The nonlinear effect can be additionally easily changed by switching polarization of light and by applying external fields. The obtained results show a possibility of existence of stable self-collimated waves (spatial solitons), existence of optical bistability in a directional coupler 
structure and nonlinear transmission of the guided modes. These types of behavior, explained as a result of the orientational effect in nematic liquid crystals, are different to those observed in waveguides with other types of optical nonlinearities (among others obtained in Kerr-type nonlinear media, in photorefractive media, in the second harmonic generation process).

\section{Acknowledgments}

This work was partially supported by the Committee for Scientific Research under the grant No. 8T11D 01915.

\section{References}

[1] I.C. Khoo, S.T. Wu, Optics and Nonlinear Optics of Liquid Crystals, World Scientific Publ., Singapore 1993.

[2] T.R. Woliński, M.A. Karpierz, Opt. Appl. 24, 265 (1994).

[3] M.A. Karpierz, T.R. Woliński, Pure Appl. Opt. 4, 61 (1995).

[4] G. Abbate, L. De Stefano, E. Santamato, J. Opt. Soc. Am. B 13, 536 (1996).

[5] M.A. Karpierz, T.R. Woliński, M. Świłło, Mol. Cryst. Liq. Cryst. 282, 365 (1996).

[6] M.A. Karpierz, M. Sierakowski, M. Świłło, T.R. Woliński, Mol. Cryst. Liq. Cryst. 320, 157 (1998).

[7] G.I. Stegeman, Opt. Appl. 26, 239 (1996).

[8] A.W. Snyder, D.J. Mitchell, Y.S. Kivshar, Mod. Phys. Lett. B 9, 1479 (1995). 